\title{
Stichproben-Verfahren in der Umfrageforschung
}

Eine Darstellung für die Praxis

Herausgegeben von

ADM Arbeitskreis Deutscher Markt- und Sozialforschungsinstitute e.V.

AG.MA Arbeitsgemeinschaft Media-Analyse e.V.

Leske + Budrich, Opladen 1999

Tochitrhe Univarsität Darmstadt

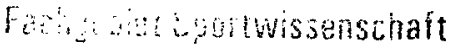




\section{Inhalt}

Vorwort der Herausgeber ADM und AG.MA

Über dieses Buch (Ute Löffler)

1 Ein Kurz-Überblick über die gebräuchlichsten Stichproben-

Verfahren in der Marktforschung (Ute Löffler)....................... 17

$1.1 \quad$ Nichtzufällige Stichproben ................................................. 18

1.1.1 Willkürliche Auswahl ...................................................... 18

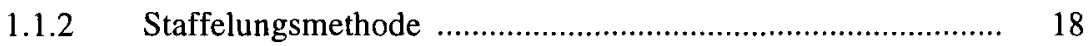

1.1.3 Bewußte Auswahl ........................................................ 18

1.2 Zufällige Stichproben bzw. Random-Stichproben ................. 19

1.2.1 Uneingeschränkte Zufallsauswahl (Unrestricted Sampling) .... 19

1.2.2 Geschichtete Zufallsauswahl (Stratified Sampling) ................ 20

1.2.3 Klumpenauswahl (Cluster Sampling) ..................................... 20

1.2.4 Mehrstufige Auswahlverfahren (Multistage Sampling) .......... 21

2 Allgemeine Theorie von Random-Stichproben

(Christian von der Heyde) .................................................... 23

$2.1 \quad$ Grundgesamtheit ............................................................. 23

2.1.1 Räumlicher Definitionsteil ................................................. 23

2.1.2 Sachlicher Definitionsteil ................................................. 23

2.1.3 Zeitlicher Definitionsteil .................................................... 24

2.1.4 Adäquationsproblem ....................................................... 24

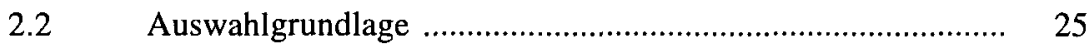

2.2.1 Adäquation von Auswahlgrundlagen …............................... 25

2.2.2 Einwohner-Melderegister .............................................. 26

2.3 Gütekriterien für Stichproben ............................................ 27

2.3.1 Repräsentativität ............................................................. 27

2.3.2 Genauigkeit .............................................................. 29

2.3.3 Präzision ..................................................................... 29 
3 Techniken und Möglichkeiten der Realisierung von RandomStichproben (Christian von der Heyde) ................................. 35

3.1 Reine Zufallsstichproben/Uneingeschränkte Zufallsauswahl .... 35

3.1.1 Geschichtete Stichproben ............................................... 37

3.1.1.1 Schichtung a priori ............................................................. 37

3.1.1.2 Schichtung a posteriori ….................................................. 39

3.2 Mehrstufige Auswahlverfahren .......................................... 40

3.2.1 Mehrstufige geschichtete Stichproben ................................ 42

3.2.1.1 Stichprobenanlage ........................................................ 42

3.2.1.2 Fehlerschätzung .............................................................. 44

3.3 Proportionale und disproportionale Stichproben ................... 45

3.3.1 Beispiele für disproportionale Stichproben ............................ 47

$3.4 \quad$ Bedeutungsgewicht ..................................................... 48

$3.5 \quad$ Allokation ..................................................................... 50

$3.6 \quad$ Gewichtung ............................................................. 54

3.7 Stichprobengröße .............................................................. 56

3.7.1 Mindestgröße bei Messung von Anteilswerten ...................... 57

3.7.2 Mindestgröße bei Messung von Durchschnitten .................... 58

$4 \quad$ Die Historie der ADM-Stichproben (Ute Löffler) ................... 61

4.1 Von den 50er und den 60er Jahren ...................................... 61

4.2 Die 70er Jahre .............................................................. 63

4.3 Die 80er Jahre ...................................................................... 64

4.4 Die 90er Jahre ................................................................... 66

5 Aufbau des ADM-Stichproben-Systems

(Kurt Behrens/Ute Löffler) ................................................. 69

5.1 Konzept der Flächenstichprobe auf Basis der Wahlbezirke .... 70

5.2 Verwendete Basisdateien .................................................. 70

5.2.1 Bundestagswahldatei ….................................................. $\quad 70$

5.2.2 Begehungsunterlagendatei .............................................. 70

5.2.3 Mikrozensus '95 …....................................................... 72

5.2.4 Gemeindedatei, Stand 30.06.1996 .................................... 73

5.3 Mögliche Grundgesamtheiten ........................................... 73

5.4 Auswahleinheiten ......................................................... 74

5.4.1 Erste Auswahlstufe: Wahlbezirke (= Sample Points) .............. 74 
5.4.1.1 Synthetisierung von Wahlbezirken ..................................... 75

5.4.1.2 Schichtung .......................................................................... 75

5.4.1.3 Ziehung von Sample Points ............................................. 76

5.4.1.4 ADM-Stichprobennetze .................................................. 77

5.4.2 Zweite Auswahlstufe: Haushalte …………………............. 78

5.4.3 Dritte Auswahlstufe: Befragungsperson ............................ 81

5.5 Regionalklassifizierungen im ADM-Stichproben-System ....... 85

5.6 Ausschöpfung bevölkerungsrepräsentativer

Random-Untersuchungen .................................................. 87

5.6.1 Qualitätsneutrale Ausfälle ............................................... 88

5.6.2 Relevante Ausfälle ..................................................... 89

5.6.3 Ausschöpfungsquote ......................................................... 89

$6 \quad$ Random-Telefon-Stichproben (Gerd Meier) …….................. 93

6.1 Vorteile von Telefonstichproben .......................................... 93

6.2 Telefondichte .................................................................... 94

$6.3 \quad$ Stichprobenziehung ..................................................... 94

.3.1 Erste Auswahlstufe - Telefonnummern bzw. Haushalte ........ 95

6.3.1.1 Auswahl aus Telefonverzeichnissen ................................... 96

6.3.1.2 Auswahl mit zufällig generierten Telefonnummern ............... 97

6.3.2 Zweite Auswahlstufe - Auswahl innerhalb des Haushalts ...... 99

6.4 Ausschöpfungsstatistik ................................................. 100

$7 \quad$ Quoten-Stichproben (Gerd Meier/Jochen Hansen) ............... 103

7.1 Anforderungen an Quotenstichproben .............................. 106

7.2 Quotenkontrolle ............................................................... 107

7.3 Zusammenfassende Bewertung ........................................ 111

$8 \quad$ Sonder-Stichproben (Christian von der Heyde) ..................... 113

$8.1 \quad$ Zeitintervallstichproben ................................................. 113

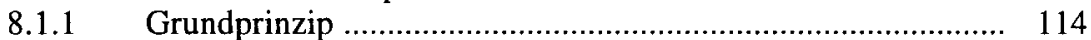

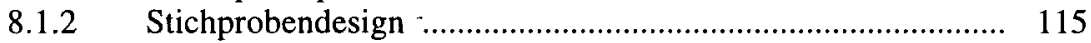

8.1.2.1 Erste Auswahlstufe: Befragungsorte ................................. 115

8.1.2.2 Zweite Auswahlstufe: Zeitintervalle .................................... 116

8.1.2.3 Dritte Auswahlstufe: Besucher .......................................... 116

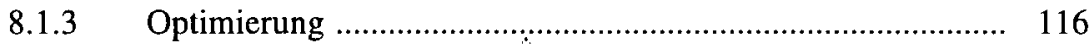

8.1.4 Kontrolle ................................................................ 118

8.2 Einwohnermeldeamts-Stichproben (EWA-Stichproben) ........ 119

8.2.1 Vor- und Nachteile ......................................................... 119

8.2.2 Stichprobendesign ..................................................... 121 
8.2.2.1 Grundgesamtheit .......................................................... 121

8.2.2.2 Erste Auswahlstufe ........................................................ 122

8.2.2.3 Zweite Auswahlstufe .................................................. 122

Anhang

9 Abgrenzungen von BIK-Stadtregionen und BIK-

Verflechtungsgebieten in den westlichen Bundesländern

der Bundesrepublik Deutschland

(Kurt Behrens/Wolfgang Marhenke)

$9.1 \quad$ Vorbemerkung .............................................................. 125

9.2 Boustedt-Modell .......................................................... 126

9.3 Räumlicher Strukturwandel f......................................... 128

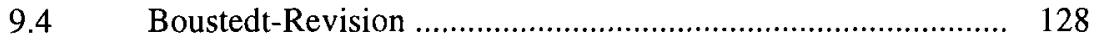

9.5 Definition von BIK-Stadtregionen ................................... 129

9.5.1 Vorgehensweise/Methodengerüst ...................................... 129

9.5.2 Pendlerstromanalyse zur Bestimmung und äußeren

Abgrenzung von Stadtregionen .......................................... 131

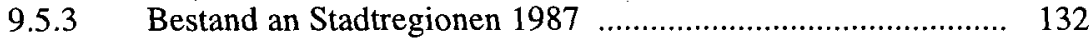

9.5.4 Innere Differenzierung der Stadtregionen .......................... 133

9.5.5 Merkmalsausprägungen nach Strukturtypen ........................ 134

9.6 BIK-Verflechtungsgebiete .......................................... 135

9.6.1 BIK-Verflechtungsgebiete in Baden-Württemberg ............... 136

10 Abgrenzungen der BIK-Stadtregionen in den östlichen Bundesländern (Kurt Behrens) ........................................... 139

$10.1 \quad$ Ansatz ............................................................... 139

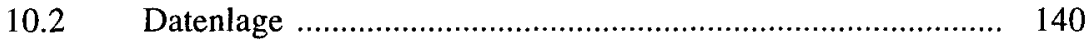

10.3 Gemeinde- und Bevölkèrungsverteilung nach Größenklassen ............................................................ 140

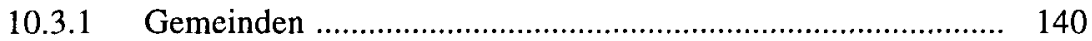

10.3.2 Bevölkerung ............................................................ 141

10.4 Besonderheiten ............................................................ 142

$10.5 \quad$ Siedlungsstrukturtypisierung ......................................... 142

10.6 Äussere Abgrenzung ....................................................... 144

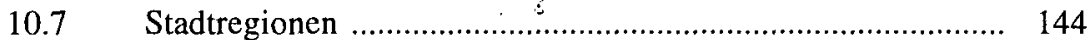

10.7.1 Regionale und statistische Verteilung ................................. 144

10.7.2 Bevölkerungsdichte ...................................................... 146

$10.8 \quad$ Zusammenfassung .......................................................... 146 


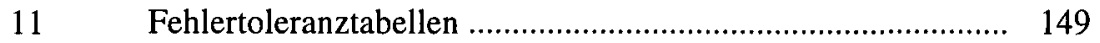

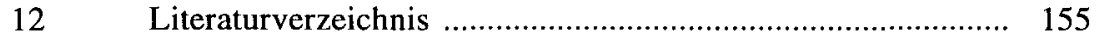

13 Über die Herausgeber ........................................................... 159

13.1 Geschichte, Aufgaben und Ziele des Arbeitskreises Deutscher Markt- und Sozialforschungsinstitute e.V. (ADM)

13.2 Geschichte, Struktur und Ziele der Arbeitsgemeinschaft Media-Analyse e.V. (AG.MA) ........................................... 160

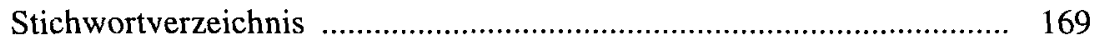

Die Autorin und Autoren .................................................................. 176

Technischer Hinweis zur Nomenklatur von im Buch verwendeten Formeln: Großbuchstaben $(N, X, P \ldots)$ bezeichnen Werte der Grundgesamtheit Kleinbuchstaben $(n, x, p \ldots)$ bezeichnen Werte der Stichprobe 